\title{
Hepatic artery anastomosis in liver transplantation
}

\author{
Siew-Weng $\underline{\operatorname{Ng}}{ }^{1}$ FRCS (Edin)
}

Liver transplant is the definitive therapy for end-stage liver failure. The challenges include overcoming technical difficulties of the transplant surgery followed by medical therapy to prevent organ rejection. The most important surgical hurdle is the prevention of hepatic artery thrombosis (HAT) that can occur in up to $9 \%$ of cases. ${ }^{1,2}$

The native liver is able to tolerate significant ischaemia with rich collateral supply but the biliary system is primarily supplied by the hepatic artery. HAT in the transplanted liver is poorly tolerated with graft loss and more than 50\% mortality and a 75\% incidence of retransplantation. ${ }^{3}$ Jain et al. noted that in 4,000 consecutive liver transplants at the University of Pittsburgh, one third of retransplantations was caused by HAT and technical failure was the main reason. ${ }^{4}$ Early detection and prevention of HAT is most dependent on microvascular surgical techniques and the regular use of Doppler ultrasound screening. ${ }^{5}$

In Singapore, at least $30 \%$ of liver transplant involves living donors due to a low donation rate. ${ }^{6}$ Compared with deceased donor liver transplantation (DDLT), living donor liver transplantation (LDLT) is associated with higher incidences of hepatic artery thrombosis, thus increasing the risk of morbidity and mortality. The donor vessels in LDLT are usually smaller and shorter, thereby increasing technical difficulty. ${ }^{7}$ To address this, a collaborative effort between the transplant surgeon and plastic surgery team at the Singapore General Hospital was established. There was a lower incidence of shortand long-term arterial complications, especially in LDLT when the plastic surgery team performed arterial anastomosis under microscopy. ${ }^{6}$

In this issue of the Annals, Tan et al. reviewed 51 consecutive cases of liver transplantation (31 cases of DDLT and 20 cases of LDLT) at the Singapore General Hospital from January 2015 to December $2018 .^{8}$ The mean age was 58 years and the most common indications for liver transplantation were non-alcoholic steatohepatitis, alcoholic liver disease and chronic hepatitis. A transplant surgeon performed the initial anastomoses of hepatic and portal veins. This was followed by arterial anastomoses under microscopy (10x magnification) by a pair of plastic surgeons. The transplant team then completed the transplant with biliary reconstruction. Serial postoperative ultrasound examinations were done on day $1,3,5,7,9$ and 14 to assess patency of the hepatic artery and biliary reconstruction.

A total of 61 arterial anastomoses were performed. There was 1 incident of hepatic thrombosis that was salvaged successfully with a radial artery interpositional graft. The hepatic arterial anastomosis was fraught with difficulties: small and short donor vessels (especially in LDLT); poor vessel quality; mismatch between donor and recipient vessel size; and microsurgery in deep recess worsened by respiratory movements. The authors outlined the technical challenges faced and illustrated the various techniques used to improve outcome for the important step of arterial reconstruction in liver transplantation.

In general, the planning of arterial anastomosis need careful consideration of (1) choice of recipient vessels; (2) methods to overcome vessel length deficiency; (3) careful preparation of poor quality vessels; and (4) meticulous techniques for anastomosis.

Choice of recipient vessels. The recipient vessel of choice is the right or left hepatic artery. However, where there is a donor vessel size mismatch, the site of recipient arterial anastomosis would need to be more proximal. This site ranges from the hepatic artery proper or the take-off of the splenic artery all the way up to the common hepatic artery. The arterial anastomosis may also be sited upstream if there is possible damage to the hepatic artery after transarterial chemoembolisation (TACE), in which case the gastroduodenal artery can be used instead.

Another situation for proximal anastomosis is when 2 recipient vessels are needed to vascularise a large liver graft. This duo anastomosis configuration may involve the gastroduodenal and common hepatic arteries. In an extreme situation of HAT salvage, revascularisation using the right gastroepiploic artery has been described. ${ }^{9}$

\footnotetext{
${ }^{1}$ Sweng Plastic Aesthetic and Reconstructive Surgery, Farrer Park Hospital, Singapore

Correspondence: Dr Siew-Weng Ng, Sweng Plastic Aesthetic and Reconstructive Surgery, Farrer Park Hospital, 1 Farrer Park Station Road, \#16-08 to 10 Connexion, Singapore 217562.

Email: sweng8@gmail.com
} 
Options for short vessels. Vessel length deficiency may be encountered in LDLT or in HAT salvage after thrombectomy and trimming of vessels. An arterial or venous interpositional vessel graft may serve as a vascular funnel where vascular inflow and outflow mismatch.

Venous grafts (e.g. from the saphenous or common iliac vein) tend to be thinner and dilate postmanipulation. A thrombosis rate of up to $23.8 \%$ has been reported, ${ }^{10}$ with long-term patency rate of less than $50 \%$ at 10 years. ${ }^{11}$ However, vein grafts have also been reportedly used with good effects in head and neck arterial reconstruction, ${ }^{12}$ as well as severe vasospastic condition. ${ }^{13}$ Arterial grafts are generally preferred for better patency rates - up to $83 \%$ at 5 years in coronary bypass has been reported. ${ }^{14,15}$ The radial artery interpositional graft is a common choice. The descending branch of lateral circumflex femoral artery can be used for patients with an abnormal Allen's test result. ${ }^{16}$ This option provides a long length and possible Y-shape configuration for double anastomoses.

The need to use interpositional graft, which increases the number of anastomoses, needs to be weighed against the increased risk of early HAT. ${ }^{17}$ This risk is increased by twofold for every extra anastomosis.

Preparation of poor quality vessels. The hepatic vessels tend to be of poor quality in liver transplantation. They can be fibrotic from previous peritonitis, or atherosclerotic related to a fatty liver, or fragile after TACE. Tan et al. outlined the steps taken to prepare the vessels for anastomosis in a deep recess using customised instruments. The vessels were trimmed using a number 11 blade held by a specially angled blade holder, on a custom-bent angulated platform for support. It was important not to skeletonise the adventitia.

Anastomotic techniques. Several important pointers were discussed by the authors to overcome the problem of friable vessels with a tendency to tear or delaminate. The anastomosis was done with $8 / 0$ suture at varying distances from the vessel edge. The staggered suturing pattern decreased the risk of circumferential tear of friable vessels. If there was a tendency for endothelial delamination, the stitching was done inside-out with the needle tagging against the endothelium downwards, rather than lifting off if the needle entered from outsidein. If both recipient and donor vessels had a tendency to delaminate, every stitch was done in the inside-out manner, thus using a single strand of dual-ended suture for each stitch of the anastomosis. The adventia was then closed with $9 / 0$ suture to improve the seal. Fibrin glue was then sprayed over and around the anastomosis, akin to having a sealant to set the vessels in the desired orientation to optimise the lie and prevent any kinking.

The discussed methodologies are essential for good vessel coaptation. The anastomosis should be watertight with no intimal damage or exposure, tension-free with no length redundancy, laid in a manner to avoid kinking or compression of vessels (by the biliary system).

Further development. With the advent of supermicrosurgical techniques for vessels less than $1 \mathrm{~mm}$ in diameter, it may be feasible to anastomose very small donor vessels to the recipient hepatic arterial system in an end-to-side manner. ${ }^{18}$ This may require special double-ended short strands of fine sutures, possibly using intraoperative indocyanine green angiography to verify patency.

Further improvement in liver transplantation outcome may be possible with the use of implantable Doppler to continuously monitor the status of the anastomosis. Implantable Doppler improved the lead time for identifying vascular compromise by an average of 10 hours, providing a specificity of $94.44 \%$ and positive predictive value of $66.66 \%$ with an overall accuracy of $95 \% .{ }^{19}$ In another study, the sensitivity and specificity of implantable Doppler has been reported to be as high as $100 \%{ }^{20}$ Any abnormal findings by an implantable Doppler would still require confirmation by conventional transcutaneous Doppler.

In conclusion, hepatic artery reconstitution is one of the most important and challenging steps of liver transplantation. In comparison with most free flap reconstruction surgery, the liver transplantation patient is usually in a poorer nutritional state, with a suboptimal coagulation profile. The liver graft is probably a more precious resource than the autologous musculocutaneous free flap harvested from the patient. The recipient vessel quality may be worse than those encountered in crush injury and less amenable to further cutback. There is the additional technical challenge of performing anastomosis in a deep recess, interrupted by respiratory movement. Therefore, it is exemplary to engage a team of experienced microsurgeons to organise the harvest of interpositional graft if needed, and perform the critical anastomosis to ensure the best outcome-what is often described as one-bite-of-the-cherry. These coordinated efforts are well illustrated in the article by Tan et al. with a very successful outcome. 


\section{REFERENCES}

1. Proposito D, Loinaz Segurola C, Garcia Garcìa I, et al. Assessment of risk factors in the incidence of hepatic artery thrombosis in a consecutive series of 687 liver transplantations. Ann Ital Chir 2001;72:187-205.

2. Abou Ella KA, Al Sebayel MI, Ramirez CB, et al. Hepatic artery thrombosis after orthotopic liver transplantation. Saudi Med J 2001;22:211-4.

3. Stange BJ, Glanemann M, Nuessler NC, et al. Hepatic artery thrombosis after adult liver transplantation. Liver Transpl 2003; 9:612-20.

4. Jain A, Reyes J, Kashyap R, et al. Long-term survival after liver transplantation in 4,000 consecutive patients at a single center. Ann Surg 2000;232:490-500.

5. Heaton ND. Hepatic artery thrombosis: conservative management or retransplantation? Liver Transpl 2013;19:S14-S6.

6. Tan EK, Tan BK, Fong HC, et al. Impact of microsurgical anastomosis of hepatic artery on arterial complications and survival outcomes after liver transplantation. Transplant Proc 2021; 53:65-72.

7. Okochi M, Ueda $\mathrm{K}$, Hirose $\mathrm{T}$, et al. A modified technique for hepatic artery reconstruction in living donor liver transplantation. Microsurgery 2010;30:541-4.

8. Tan BK, Fong HC, Tan EK, Raj JP. Strategies for a successful hepatic artery anastomosis in liver transplantation: A review of 51 cases. Ann Acad Med Singap 2021;50:679-85.

9. Ikegami T, Kawasaki S, Hashikura Y, et al. An alternative method of arterial reconstruction after hepatic arterial thrombosis following living related liver transplantation. Transplantation 2000;69:1953-5.

10. Tzakis AG, Gordon RD, Shaw BW Jr, et al. Clinical presentation of hepatic artery thrombosis after liver transplantation in the cyclosporin era. Transplantation 1985;40:667-71.

11. Campeau L, Enjalbert M, Lesperance J, et al. Atherosclerosis and late closure of aortocoronary saphenous vein graft: sequential angiographic studies at 2 weeks, 1 year, 5 to 7 years, and 10 to 12 years after surgery. Circulation 1983;68:II1-117.

12. Koshima I, Yamamoto H, Hosoda M, et al. Free combined composite flaps using the lateral circumflex femoral system for repair of massive defects of the head and neck regions: an introduction to the chimeric flap principle. Plast Reconstr Surg 1993;92:411-20.

13. Kim YH, Ng SW, Seo HS, et al. Classification of Raynaud's disease based on angiographic features. J Plast Reconstr Aesthet Sur 2011;64:1503-11.

14. Acar C, Jebara VA, Portoghese M, et al. Revival of the radial artery for coronary artery bypass grafting. Ann Thorac Surg 1992; 54:652-60.

15. Dietl CA, Benoit $\mathrm{CH}$. Radial artery graft for coronary revascularization: technical considerations. Ann Thorac Surg 1995;60:102-110.

16. Imakuma ES, Bordini AL, Millan LS, et al. Comparative morphometric analysis of 5 interpositional arterial autograft options for adult living donor liver transplantation. Transplant Proc 2014;46:1784-8.

17. Piscaglia F, Vivarelli M, La Barba G, et al. Analysis of risk factors for early hepatic artery thrombosis after liver transplantation. Possible contribution of reperfusion in the early morning. Dig Liver Dis 2007;39:52-9.

18. Iida T, Yoshimatsu H, Yamamoto $\mathrm{T}$, et al. A pilot study demonstrating the feasibility of supermicrosurgical end-to-side anastomosis onto large recipient vessels in head and neck reconstruction. J Plast Reconstr Aesthet Surg 2016;69:1662-8.

19. Vasant Kulkarni S, Rao PP, Naidu CS, et al. Evaluation of implantable Doppler probe continuous monitoring of hepatic artery anastomosis after liver transplantation. Med J Armed Forces India. 2021;77:349-54

20. Guillemaud JP, Seikaly H, Cote D, et al. The implantable CookSwartz Doppler probe for postoperative monitoring in head and neck free flap reconstruction. Arch Otolaryngol Head Neck Surg 2008;134:729-34. 